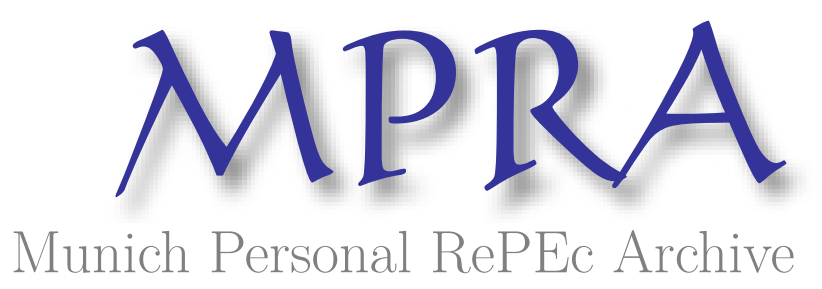

\title{
Not so cheap talk: Costly and discrete communication
}

Hertel, Johanna and Smith, John

Rutgers University-Camden

28 June 2010

Online at https://mpra.ub.uni-muenchen.de/23560/

MPRA Paper No. 23560, posted 29 Jun 2010 19:34 UTC 


\title{
Not So Cheap Talk: Costly and Discrete Communication*
}

\author{
Johanna Hertel and John Smith ${ }^{\dagger}$
}

June 28, 2010

\begin{abstract}
We model an interaction between an informed sender and an uninformed receiver. Like the classic cheap talk setup, the informed player sends a message to an uninformed receiver who is to take an action which affects the payoffs of both players. However, unlike the classic cheap talk setup, the sender can communicate only through the use of discrete messages. In particular, the sender has a finite set of message elements with which to compose messages. The sender incurs a communication cost which is increasing in the number of elements contained in the message. We characterize the resulting equilibria with a permissive out-of-equilibrium restriction. We introduce a stronger out-of-equilibrium requirement and show that if the sender and receiver have aligned preferences regarding the action of the receiver then only the most informative equilibrium exists. When the preferences between players are not aligned, we show that our stronger condition does not guarantee uniqueness and we provide an example where an increase in communication costs can improve communication. As we show in an example, this improvement can occur to such an extent that an equilibrium can outperform the Goltsman et. al. (2009) upper bound for payoffs in mediated communication.
\end{abstract}

Keywords: information transmission, cheap talk, costly communication JEL: C72, D82, D83

*The authors would like to thank Andreas Blume, Gabor Virag, and participants at SING5 in Amsterdam, the SABE/IAREP conference in Halifax, the 20th International Festival of Game Theory in Stony Brook, the Workshop on Behavioral Game Theory in Stony Brook, EEA/ESEM in Barcelona, Behavioral and Quantitave Game Theory Conference in Newport Beach and the Midwest Theory Conference at Northwestern for helpful comments. This research was supported by Rutgers University Research Council Grants \#202344 and \#202084. Portions of this paper were previously circulated under the title "Not So Cheap Talk: A Model of Advice with Communication Costs."

${ }^{\dagger}$ Corresponding Author: Rutgers University-Camden, Department of Economics, Email: smithj@camden.rutgers.edu, Website: http://crab.rutgers.edu/ smithj/ 


\section{Introduction}

A person will often use words to convey information about a complex and nuanced reality. Words are discrete objects in the sense that their properties are very different from that of real numbers. Statements are then finite combinations of these discrete objects. Although there is rarely a single word to convey the entire extent of the complex reality, the communicator can approach this complexity by constructing more elaborate and complex statements.

In this paper, we take the view that communication is necessarily discrete. We analyze the implications of such discrete communication in a strategic interaction between an informed sender and an uninformed receiver. In our model, the sender learns the state of the world on the unit interval and transmits a discrete message to the receiver. After observing the message, the receiver is to take an action which affects the payoffs of both sender and receiver.

In our model, the sender can send either a costless, empty ${ }^{1}$ message $(\varnothing)$ or a costly, nonempty message which is constructed from a set of message elements $\left\{e_{1}, \ldots, e_{\gamma}\right\}$. We assume that the cost of transmitting a message is an increasing function of the number of elements in the message. For instance, the cost of transmitting the empty message is $c(0)$ and the cost of transmitting a message with three elements, say $\left(e_{3}, e_{5}, e_{2}\right)$, is $c(3)$. In our model, there is no syntax and the message elements have no a priori meaning. We view this a relatively simple and general way to model discrete communication in which more elaborate messages can be constructed but at a cost to the sender.

We characterize the resulting equilibria under a permissive out-of-equilibrium condition, which we call Condition $P$. This condition roughly states that if a receiver ever observes an out-of-equilibrium message then the receiver believes that the message was sent by the sender with the least incentive to deviate. We characterize the equilibria under Condition $P$. Like the original cheap talk paper of Crawford and Sobel (1982) (hereafter referred to as CS), this condition implies many equilibria.

We introduce a stronger out-of-equilibrium restriction on beliefs, Condition $L$. This restriction roughly states that if a receiver ever observes an out-of-equilibrium message then the receiver believes that the message was sent by the sender with the most incentive to deviate. We show that under Condition $L$, if there is perfect alignment between the preferences over the receiver's action and we restrict attention to pure strategies of the sender then the equilibria is the one preferred by the receiver. This result is similar to that when the No Incentive to Seperate $($ NITS $)$ condition is applied to the original cheap talk model as in Chen, Kartik and Sobel (2008).

Further, if preferences for the receiver's action are not aligned, we show that Condition $L$ does not guarantee a unique equilibrium and we provide an example where an increase in communication costs improves communication. Quite surprisingly, when preferences are not aligned and the sender incurs communication costs there exists equilibrium in which the

\footnotetext{
${ }^{1}$ Throughout the paper we describe the costless message as empty rather than the condition of having not received a message. This is because, in a practical setting, it might not be easy to distinguish between the sender having decided to not send a message and the sender having not yet sent a message. To rule out this confusion we describe the costless message as empty.
} 
receiver's payoffs outperform the Goltsman et. al. (2009) upper bound for payoffs in such communication games. We provide an example of such an equilibrium.

\section{Related Literature}

\subsection{Cheap Talk and Related Models}

The large strand of cheap talk literature was initiated by CS. The authors show that for mild differences in the preferences of receiver and sender, meaningful, albeit incomplete, communication can occur. ${ }^{2}$ CS shows that equilibrium always takes the form that the state space is partitioned and the messages are sent such that a unique action is induced within each element of the partition. Our equilibrium is analogous, in that a unique action is induced on each interval.

A number of papers have extended the original CS model. For instance, Morgan and Stocken (2003) extend the CS model to the case where there is uncertainty regarding the degree of divergence between the preferences of the sender and receiver. Fischer and Stocken (2001) model a situation where the receiver has imperfect information about the state. Blume, Board and Kawamura (2007) modify the CS setup where communication errors (or noise) can occur. In our view, the present paper shares the goal of these papers: to learn the significance of a particular assumption in the CS model. Here we seek to learn the importance of the assumption that messages are plentiful and equally costless.

Blume, Board and Kawamura (2007) demonstrated that a small amount of noise can improve communication in the CS model. In particular, the authors show that there is an optimal amount of noise which maximizes the receiver's payoffs. Relatedly, Goltsman et. al. (2009) study general communication in the CS model. The authors study mediation, whereby a neutral third party or mediator will advise both of the players. Surprisingly, Goltsman et. al. (2009) find that the payoffs in the equilibrium with the optimal amount of noise found by Blume, Boad and Kawamura is the the upper bound of payoffs for the receiver in any mediated communication in the CS setting. We provide an example of an equilibrium in which communication costs imply that the receiver can attain a payoff above this upper bound.

The original CS model exhibits a large number of possible equilibria. Specifically, CS shows that for a given difference in the preferences of the sender and receiver, if there is an equilibrium where the state space is partitioned into a finite number of partitions (say $n$ ) then there are equilibria which partition the state space into $1,2, .$. and $n-1$ elements. Our out-of-equilibrium Condition $P$ leads to a similar result in that, for a given set of parameter values and an ordering of messages, there exists a message within the ordering which has a maximum complexity (say $i$ ) which could which could constitute an equilibria. Additionally, under Condition $P$ there are equilibria where the complexity of this message could take all available values between 1 and $i-1$.

\footnotetext{
${ }^{2}$ Spector (2000) shows that in the CS model, as the difference between the preferences of sender and receiver converge, the equilibrium converges towards full information transmition.
} 
As is often the case for multiple equilibria, researchers have sought to reduce the number of cheap talk equilibria through refinements. ${ }^{3}$ A recent innovation in this regard is the Condition No Incentive to Seperate (NITS) of Chen, Kartik and Sobel (2008). NITS restricts attention to equilibria in which it is not the case that the sender who receives the state $s=0$ (with a state space of $[0,1])$ prefers to perfectly reveal the state. In their Proposition 3 , the authors show that if the monotonicity condition holds in the CS model (as it does in the commonly used "uniform-quadratic" case) then NITS selects a unique equilibrium which is the most informative, i.e. contains the largest possible number of partitions. Our Condition $L$ is similar to NITS in that if an out of equilibrium message is observed then the receiver believes that the state is equal to zero. ${ }^{4}$ And similar to NITS, Condition $L$ for the case of aligned preferences rules out each equilibrium except those in the most informative class. However, we also provide an example that, when preferences are not aligned, Condition $L$ does not guarantee uniqueness.

\subsection{Costly Communication}

We are not the first to introduce costly communication into the CS model. Austen-Smith and Banks (2000) and Kartik (2007) model costly and costless messages in the original CS model. ${ }^{5}$ While the present paper shares the feature that communication is costly, we differ in that here communication is discrete and increasingly costly in the elaborateness of the message.

In Dewatripont and Tirole (2005) the sender incurs costs of effectively communicating information and the receiver incurs costs in absorbing information. In Dewatripont and Tirole, information is either understood or not. ${ }^{6}$ By contrast, the states in our model are better characterized by the degree to which they are learned. Additionally, in Dewatripont and Tirole the sender and receiver necessarily have different preferences over the action of the receiver. By contrast, we examine both the cases where they are aligned and are unaligned. ${ }^{7}$

To our knowledge, there are two costly communication papers in which there are shades of understanding. In Calvo-Armengol, de Marti and Prat (2009) the sender transmits a necessarily noisy signal but can affect its precision by a incurring larger communication cost. In our view, this assumption is less appropriate when modeling complex communication as the signal actually sent is not necessarily less complex than the sender's most preferred signal. In

\footnotetext{
${ }^{3}$ For instance, see Banks and Sobel (1987), Cho and Kreps (1987), Farrell (1993), Kohlberg and Mertens (1987), Matthews, Okuno-Fujiwara and Postlewaite (1991).

${ }^{4}$ Note that the formal statement of NITS relates to the payoffs of the receiver. Nevertheless, implicit in the statement of NITS is that after observing an out-of-equilibrium message, the receiver believes with certainty that the state is zero. We focus on the beliefs associated with the out-of-equilibrium message, therefore our statement of Condition $L$ specifies beliefs.

${ }^{5}$ The cost of these messages are unrelated to the unknown state of the world. See Spence (1973) for the classic model of the case where the cost of transmitting a signal varies with the underlying state of the world. Also see Gossner, Hernandez and Neyman (2006).

${ }^{6}$ See Austen-Smith (1994) for another costly communication paper in which information is either understood or not.

${ }^{7}$ Also note that we are not the first to model communication between a sender and receiver who have identical preferences over the receiver's action. For instance, Morris (2001) presents such a model in which, due to reputation effects, the sender might not truthfully reveal the state of the world. Also see Blume and Board (2009), Che and Kartik (2009), Cremer, Garicano and Prat (2007) and Jager, Koch-Metzger and Riedel (2009).
} 
Cremer, Garicano and Prat (2007) a fixed number of partition elements are optimally arranged in order to minimize communication problems between an informed sender and an uninformed receiver who have identical preferences over the action of the receiver. Like Cremer et. al., we find that the equilibrium mapping from the state space to the message space is not uniform. In Cremer et. al. this is due to the differential likelihood of events and in our paper it is due to the differential cost of the available messages.

Our model makes the prediction that the sender will be silent over some states of the world. Similarly, the discretionary-based disclosure literature ${ }^{8}$ finds the conditions under which a manager would withhold information about the value of the firm. For instance, Verrecchia (1983) shows that in the presence of disclosure costs, there are states for which the manager will chose not to disclose information. We differ from this literature in the sense that we consider the case where sender and receiver have identical preferences over the action of the receiver. Therefore, to our knowledge we are the only paper which finds silence even in the case of perfect alignment of preferences.

\section{Model}

A sender $(S)$ and receiver $(R)$ play a communication game in a single period. Payoffs for both players depend on the receiver's action $a$, as well as the state of the world $s$. A state is an element of the closed interval $[0,1]$. The receiver's action space is $\mathbb{R}$. The receiver's utility from action $a$ when the state is $s$ is:

$$
u^{R}(a, s)=-(a-s)^{2} .
$$

The receiver has ex-ante beliefs that the state is uniformly distributed on $[0,1]$. The sender observes the state and can communicate some information about the state to $R$ by sending a message $m$ where $m \in \mathcal{M}$. Each $m$ is composed of message elements $\left\{e_{1}, \ldots, e_{\gamma}\right\}$ where $\gamma \geq 1$. If we let $\left\{e_{1}, \ldots, e_{\gamma}\right\}^{k}$ represent the set of all $k$-tuples of elements then we write $\mathcal{M}=\varnothing \cup\left(\cup_{k=1}^{\infty}\left\{e_{1}, \ldots, e_{\gamma}\right\}^{k}\right)$. We interpret message $m$ as more complex than $m^{\prime}$ if $m$ has more elements than $m^{\prime}$. The cost of communication $(c: \mathbb{N} \Rightarrow \mathbb{R})$ is an increasing function of the number of elements in the message. ${ }^{9}$ Further, if message $m^{i}$ contains $i$ elements and message $m^{i+1}$ contains $i+1$ elements then we require that $c(i+1)-c(i) \geq \psi>0$. We also assume that $c(0)=0$. In a slight abuse of notation, we will refer to the case described above as $c>0$, the case where there is no communication costs as $c=0$ and the case of both communication costs and the absence of communication cost as $c \geq 0 .^{10}$ The sender's utility is:

$$
u^{S}\left(a, m^{i}, s\right)=-(a-s-b)^{2}-c(i)
$$

where $b \geq 0$.

The sender's strategy is $\mu:[0,1] \rightarrow \mathcal{M}$ and the receiver's strategy is $\alpha: \mathcal{M} \rightarrow \mathbb{R}$. Note

\footnotetext{
${ }^{8}$ See Verrecchia (2001) for a review of the discretionary-based disclosure literature.

${ }^{9}$ Also see Vartiainen (2009) for a similar notion of communication costs.

${ }^{10}$ Of course, since there is no outside option, adding any amount to the function $c$ would not affect our results. We assume that $c(0)=0$ in order to render meaningful our notation of $c=0$ and $c>0$.
} 
that we restrict attention to pure strategies for both sender and receiver. ${ }^{11}$ We seek an equilibrium $\left(\mu^{*}, \alpha^{*}\right)$ such that $S$ chooses the optimal action, given accurate beliefs $R$ chooses the optimal action and $R$ 's beliefs are derived from Bayes' Rule wherever possible. We denote $R$ 's beliefs as $\beta(s \mid m)$.

Definition 1 For an equilibrium $\left(\mu^{*}, \alpha^{*}\right)$ we require:

$$
\begin{gathered}
\text { for each } s \in[0,1], m=\arg \max _{m^{\prime}} u^{S}\left(\alpha^{*}\left(m^{\prime}\right), m, s\right) \\
\text { for each } m \in \mathcal{M}, \alpha(m)=\arg \max _{a^{\prime}} \int u^{R}\left(a^{\prime}, s\right) \beta(s \mid m) d s
\end{gathered}
$$

and that $R$ 's beliefs are derived from $S$ 's strategy.

Before we proceed to the results, we briefly discuss some of our modeling choices. The state space is designed to be richer than the message space ${ }^{12}$ as the state space is uncountably infinite and there are only a finite number of messages which can be transmitted with a finite cost. We believe that this captures an important aspect of reality: it is impossible to completely communicate the complexity of the real world, one may only increase the precision of communication by expending more costly effort.

Also, we assumed that there is only a single empty message. This is because it would seem to be the case that there is only one way to literally say nothing. However, if there were a finite number of different ways to say nothing then the qualitative results which follow would not be changed.

Perhaps a natural questions is, why not model communication which is necessarily noisy where the sender incurs a communication cost which is decreasing in the variance of the possible messages. Within this possibility, there arise some features which we find unappealing.

First, suppose that the sender would costlessly specify the preferred action of the receiver and the preferred amount of communication costs to a third party who would then add the appropriate amount of noise to the message. As Blume, Board and Kawamura (2007) showed, communication is enhanced by at least a small amount of noise. It would seem to violate the spirit of the model that, even if communication is costless, the sender would prefer to transmit a message with noise. Further, this problem is not avoided if the noise is determined by the amount of effort expended by the sender. Therefore, we do not view this possibility as an adequate substitute for our modeling choices.

As a second option, suppose that the sender would costlessly specify the upper and lower bound of the possible states which is communicated to the receiver. We argue that it should be viewed as less costly to send a message indicating that the state is in the interval, $[0.2,0.4]$ than in the interval [0.2132735409, 0.4132735409], as the latter would take more time to specify.

\footnotetext{
${ }^{11}$ The restriction to pure strategies on the part of the receiver is without loss of generality. However, the restriction on the part of the sender is not entirely without loss of generality. Lemma 9 and the related discussion in the appendix clarifies the extent to which the restriction to pure strategies is without loss of generality.

${ }^{12}$ This assumption also appears in Jager, Koch-Metzger and Riedel (2009) and Lipman (2006).
} 
This option seems to betray the principle that more elaborate messages should be more costly to send. Further, we would have to assume that the receiver is unsophisticated. For instance, if the sender wished to communicate the state, $s=0.315789215$, the sender could send the message leading to the possible interval $[0.315789215,1]$ and the sophisticated receiver would know that the state is certain to be 0.315789215 . To avoid these types of problems, we would either have to model the receiver as unsophisticated or to model communication as we do here. In our view, modeling complexity is best done with a minimal abstraction of the nature of the complexity, otherwise it is all too easy to retain some features of a model where all messages are plentiful and equally costly.

In both of the above options, the communication does not, in our view, resemble communication via words. Most notably the resulting equilibrium would be a fully separating equilibrium whereby each state would induce a unique action by the sender. By contrast, the equilibrium in our model is a pooling equilibrium in that for generic parameter values, several states induce identical actions by the sender. This seems to be more consistent with our intuition regarding communication.

Finally, note that a possible set of message elements are the set of single digit integers: $\{0$, $1,2,3,4,5,6,7,8,9\}$. Therefore, the sender has the means to truthfully disclose the exact state. However, we will see that, not only will this will not happen in equilibrium, but there are many states for which the sender transmits the empty message.

\section{Preliminaries}

Before we offer a characterization of the equilibria, we introduce some notation and provide a necessary condition for the equilibria.

Although our equilibria share some of the familiar characteristics of the cheap talk literature, the additional results which emerge will require the flexibility provided by the notation which we now define. Like the CS equilibria, messages are sent on connected, nonoverlapping intervals. ${ }^{13}$ Therefore, we may characterize an equilibrium by a set of cutoff states where we denote the number of messages used in equilibrium as $n$ by listing the order of the messages messages $m_{1}, \ldots, m_{n}$. The messages induce a set of cutoff states which we denote:

$$
0=s_{1} \leq s_{2} \leq \ldots \leq s_{h} \leq \ldots \leq s_{n} \leq 1=s_{n+1} .
$$

Equilibrium is such that $S$ 's messages are sent as intervals on the state space:

$$
m_{h}=\mu^{*}(s) \text { for } s \in\left[s_{h}, s_{h+1}\right)
$$

and $R$ best responds in a straightforward manner:

$$
\alpha^{*}\left(m_{h}\right)=\bar{a}\left(s_{h}, s_{h+1}\right)=\arg \max _{a^{\prime}} \int_{h}^{h+1} u^{R}\left(a^{\prime}, s\right) \beta(s \mid m) d s
$$

\footnotetext{
${ }^{13}$ See the appendix for the proof of Lemma 7 which shows that the equilibrium strategy for $S$ entails sending a message for states which are conntected intervals and Lemma 8 which shows that the intervals are not partially overlapping.
} 
where $\bar{a}\left(s_{h}, s_{h+1}\right)$ is the best response of $R$ if the state is known to be between $s_{h}$ and $s_{h+1}$.

Definition 1 implies the arbitrage equation, also standard in the cheap talk literature. This expression characterizes the equilibrium set of cutoff states:

$$
u^{S}\left(\bar{a}\left(s_{h}, s\right), m_{h}, s\right)=u^{S}\left(\bar{a}\left(s, s_{h+2}\right), m_{h+1}, s\right) \text { for } h \in\{1, \ldots, n-1\} .
$$

We define $\lambda_{h}$ to be the mass of states such that $m_{h}=\mu(s)$. Since the messages are sent on an interval of the state space and the states are distributed uniformly, $\lambda_{h}=s_{h+1}-s_{h}$ when $m_{h}=\mu(s)$ for $s \in\left[s_{h}, s_{h+1}\right)$ and $m_{h} \neq \mu(s)$ for $s \notin\left[s_{h}, s_{h+1}\right)$.

While subscripts refer to the order of the messages, we use superscripts to denote the complexity of the message. Therefore, we denote the empty message as $m^{0}$, a message with a single element as $m^{1}$ and so on. Correspondingly, we define $\lambda_{h}^{j}$ to be the mass of states associated with the $h$ th message which has complexity $j$. An an equilibrium in which there are $n$ actions induced will require that:

$$
\lambda_{1}+\lambda_{2}+\ldots+\lambda_{n}=1 \text { where } \lambda_{h} \geq 0 \text { for every } h \in\{1, \ldots, n\}
$$

We now provide a necessary condition for an equilibrium. Lemma 1 describes the relative the size of two adjacent intervals. Like the CS model, for $b>0$ the interval size is increasing in its location on the state space. In other words, for $b>0$ and $c=0$, the intervals representing larger numbers on the state space are larger than intervals representing smaller numbers. The lemma also shows that the size of the interval is decreasing in the cost of the signal transmitted on that interval.

Lemma 1 For any equilibrium $\left(\mu^{*}, \alpha^{*}\right)$ where $b \geq 0$ and $c \geq 0$ in which there are $n$ actions induced, it must be that:

$$
\left(\lambda_{h+1}^{j}\right)^{2}-\left(\lambda_{h}^{i}\right)^{2}=4 b\left[\lambda_{h+1}^{j}+\lambda_{h}^{i}\right]+4[c(i)-c(j)] \text { for } h \in\{1, \ldots, n-1\} .
$$

Intuitively, note that more costly signals are conserved. In particular, the cost of a signal is negatively related to the size of the state space on which it is transmitted. Also note that when $b>0$ and $c=0$, we are essentially in the CS model as expression (4) easily reduces to expression (21) in CS. Therefore, when $b>0$ and $c=0$ the intuition behind the relationship between the interval size and its location on the state space is identical to that in CS.

\section{Equilibrium Characterization under Condition $P$}

In this section we characterize the equilibrium using a permissive out-of-equilibrium specification of beliefs, Condition $P$. Condition $P$ is permissive in that it admits a great deal of equilibria. By assuming this condition, we can best glean insights from the model and highlight the utility of Condition $L$. Upon observing an out-of-equilibrium message, Condition $P$ specifies that, among the states which are equal to the actions selected by the receiver, the receiver believes that it is the state which induces the message associated with the lowest communication cost. 
Condition $P$ : Given $(\mu, \alpha)$, if there does not exist an $\widehat{s}$ such that $\mu(\widehat{s})=\widehat{m}$ and $R$ observes $\widehat{m}$ then $R$ believes that the state is certain to be, among the states such that $\alpha\left(\mu\left(s^{\prime}\right)\right)=s^{\prime}$, the $s^{\prime}$ where $c\left(\mu\left(s^{\prime}\right)\right)$ is the smallest. If there are several such $s^{\prime}$ then the sender believes that the state is the smallest of these.

In the introduction it was said that if the receiver observes an out-of-equilibrium message then Condition $P$ roughly specifies that the receiver believes that the state is such that the sender had the least incentive to deviate. Now we can better qualify that statement. The closer the alignment of preferences (smaller $b$ ) the smaller the difference between the action of the receiver and the sender's most preferred action. Therefore, the closer the alignment of preferences, the more accurately we can say that if the receiver observes an out-of-equilibrium message then Condition $P$ specifies that the receiver believes that the state is such that the sender had the least incentive to deviate.

Note that Condition $P$ implies that no new actions are induced by an out-of-equilibrium message. This fact accounts for the permissive nature of Condition $P$ : it is relatively difficult to locate a deviation from a potential equilibrium. Also note that the second statement in Condition $P$ applies only to the case where there are several states in which the payoffs to the sender are the highest. In this event, the statement ensures that there are well defined beliefs after observing an out-of-equilibrium message. The results of the paper would not be affected if we selected another tie-breaking rule.

We now characterize the equilibria under Condition $P$. Our first result is that under Condition $P$ the empty message will always be used in equilibrium. Further, for any positive communication costs there will always exist an uninformative equilibrium where the empty message is sent on all states.

Lemma 2 For any equilibrium $\left(\mu^{*}, \alpha^{*}\right)$ under Condition $P$, there will exist a state $s$ such that $\mu^{*}(s)=m^{0}$. Also for any $b \geq 0$ and $c>0$, under Condition $P$ there will exist an equilibrium $\left(\mu^{*}, \alpha^{*}\right)$ such that $\mu^{*}(s)=m^{0}$ for all $s \in[0,1]$.

Proof: Consider $\left(\mu^{*}, \alpha^{*}\right)$ in which the empty message is not used. Upon observing an out-of-equilibrium message, suppose that Condition $P$ specifies that the receiver will believe that the state is $s^{\prime}$. Therefore, a profitable deviation for $S$ is to send the empty message for $s^{\prime}$ rather than a costly message. Hence, in any equilibrium under Condition $P$ the empty message must be used. Now consider $\left(\mu^{*}, \alpha^{*}\right)$ such that $\mu^{*}(s)=m^{0}$ for all $s \in[0,1]$. All costly messages would not induce a different action from the receiver and yet the sender would incur a cost of communication. Therefore, under Condition $P$ there is no profitable deviation from this completely uninformative equilibrium.

Lemma 2 states that the empty message will always be used in any equilibrium which satisfies Condition $P$, however, we will see a stronger analogous result for Condition $L$. In particular we show that under Conditon $L$, there cannot be an equilibrium in which there are unused, cheaper messages. Lemma 2 also states that for any communication costs, under Condition $P$ there will always exist an equilibrium which is completely uninformative. Again, we will see how this changes under Condition $L$. We will show that if $b=0$, the only time 
that there can be an uninformative equilibrium under Condition $L$ is when there does not exist an equilibrium under Condition $L$ involving any costly messages.

Next, we offer a definition which summarizes the necessary conditions for an equilibrium under Condition $P$.

Definition $2 A(\mu, \alpha)$ is P-feasible if there exists a state $s$ such that $\mu(s)=m^{0}$ and satisfies expressions (1), (3) and (4).

We now demonstrate a procedure for determining the range of $(\mu, \alpha)$ which are $P$-feasible. As such, consider a candidate message $m_{h}$. We will show that for a general ordering of messages to the left of and to the right of this candidate message, there is always a largest candidate message such that all such candidates messages with lower costs are $P$-feasible and all messages with higher costs are not $P$-feasible.

Consider an ordered $h-1$ tuple of messages $\left(m_{1}, \ldots, m_{h-1}\right)$ where $h \geq 1$, which we denote as $m_{L}$. These are the messages which are sent on states which are lower than (or to the left of) our candidate message $m_{h}$. Also consider an ordered $n-h-1$ tuple of messages, where $n-h \geq 1$. In a slight abuse of notation, this tuple can be written as $\left(m_{h+1}, \ldots, m_{n}\right)$ which we denote as $m_{R}$. These are the messages which are sent on states that are higher than (or to the right of) the candidate message $m_{h}$. Now consider the aggregation of these messages in an ordered tuple $\left(m_{L}, m_{h}^{k}, m_{R}\right)$. We will vary composition of the candidate message $m_{h}^{k}$ among the the messages which are available. The messages in $\mathcal{M}$ but not used by $m_{L}$ and $m_{R}$ can be used in $m_{h}^{k}$. We increase $k$, among these remaining messages until the tuple $\left(m_{L}, m_{h}^{k}, m_{R}\right)$ is no longer $P$-feasible.

The setup described above can accommodate the case where $m_{h}$ is the first (last) message in the configuration. This is accomplished by an empty ${ }^{14} m_{L}\left(m_{R}\right)$. Also, it can be either that $m_{L}$ or $m_{R}$ contains the empty message $m^{0}$. However, it cannot be that both contain the empty message because there is only a single such message in $\mathcal{M}$. Finally, we say that $\left(m_{L}, m_{h}^{k}, m_{R}\right)$ is maximal and $P$-feasible if $\left(m_{L}, m_{h}^{k^{\prime}}, m_{R}\right)$ where $k^{\prime}>k$ is not $P$-feasible. The following lemma shows that for any $m_{L}$ and $m_{R}$ there is always a maximal and $P$-feasible ordered tuple of messages forming $(\mu, \alpha)$.

Lemma 3 For any $c>0$ and $P$-feasible configuration of messages $\left(m_{L}, m_{R}\right)$ forming $(\mu, \alpha)$ there exists a there exists a maximal, P-feasible configuration $\left(m_{L}, m_{h}^{k}, m_{R}\right)$ forming $(\mu, \alpha)$.

Note that according to Lemma 3 , the $k$ such that $\left(m_{L}, m_{h}^{k}, m_{R}\right)$ which is found to be maximal and $P$-feasible depends on $m_{L}$ and $m_{R}$. Therefore, checking the set of $P$-feasible $(\mu, \alpha)$ can be rather tedious because it must be accomplished for each feasible $\left(m_{L}, m_{R}\right)$.

We are ready to characterize the equilibria under Condition $P$. We now show that each $P$-feasible $(\mu, \alpha)$ will form an equilibrium under Contidion $P$.

Proposition 1 If $(\mu, \alpha)$ is $P$-feasible then it is an equilibrium under Condition $P$.

\footnotetext{
${ }^{14}$ Empty as in not containing a message rather than containing the costless, empty message $m^{0}$.
} 
As the above proposition suggests, there are many equilibria under Condition $P$. This abundance of equilibria stands in contrast to our results in the following section. There we show that for $b=0$, the only equilibria which satisfies Condition $L$ are, among those which satisfy Condition $P$, the equilibria which are most informative.

\section{Alignment of Preferences under Condition $L$}

Here we focus on the implications of Condition $L$ for case where the preferences regarding the receiver's actions are perfectly aligned $(b=0)$. In the sequel we analyze the implications of Condition $L$ on the case where there is imperfect alignment of preferences $(b>0)$. In this section, we ultimately show that only the most informative equilibrium exists under Condition $L$.

We begin this section by noting that when $b=0$, the order of the signals does not matter. We can rewrite expression (4) in Lemma 1 for the case of $b=0$ as:

$$
\left(\lambda^{j}\right)^{2}-\left(\lambda^{i}\right)^{2}=4[c(i)-c(j)] .
$$

As expression (5) suggests, the interval size on which a message is sent is determined only by its communication cost and not by its placement in the state space. In other words, when $b=0$ the order of the messages does not matter. As a result, expression (5) does not contain subscripts. This simplification somewhat mitigates the difficulty in identifying equilibria which satisfy Condition $P$. However, the task is still difficult because Condition $P$ only implies that the empty message is used, all other combinations of signals which are $P$-feasible are not ruled out. Now we show that Condition $L$ greatly reduces the difficulty in identifying equilibria because only a unique class of $(\mu, \alpha)$ can form an equilibrium.

If the incentives are aligned $(b=0)$ and there are $n$ actions induced in equilibrium then there are $n+1$ states in which the sender has the most incentive to deviate. ${ }^{15}$ Therefore, as a matter of convention, we select one of these $n+1$ states. Hence, Condition $L$ specifies that if an out-of-equilibrium message is observed then $R$ believes that the state is certain to be $s=0$.

Condition $L$ : Given $(\mu, \alpha)$, if there does not exist an $\widehat{s}$ such that $\mu(\widehat{s})=\widehat{m}$ and $R$ observes $\widehat{m}$ then $R$ believes that the state is certain to be $s=0$.

The introduction stated that Condition $L$ specified that if the receiver ever observes an out-of-equilibrium message then the receiver believes that the message was sent by the sender with the most incentive to deviate. Now we can better qualify that statement. The closer the alignment of preferences (smaller $b$ ), the more accurately we can say that if the receiver observes an out-of-equilibrium message then the receiver believes that the message was sent by the sender with the most incentive to deviate. Before the statement of Condition $L$, we noted that if $b=0$ and there are $n$ actions induced in equilibrium then there are $n+1$ states

\footnotetext{
${ }^{15}$ See Lemma 10 in the appendix.
} 
in which the sender has the most incentive to deviate. All of the results involving $b=0$ would follow if we selected any of the other such $n$ states. ${ }^{16}$

Condition $L$ is similar to NITS in the sense that the former specifies beliefs in the event of an out-of-equilibrium message which are implicit in the statement of the latter. Additionally, for the case that $b>0, s=0$ is typically not the state with the lowest equilibrium payoffs for the sender. For this reason we view Condition $L$ in the case of $b=0$ to be at least as reasonable as NITS in the case of $b>0$.

Before we state the main result regarding the equilibria under Condition $L$, we provide the following lemmas which are used in the proof of the result. The first lemma which we present shows that there cannot exist holes in the equilibrium. Specifically, it must be the case that if a message of a certain cost is used in equilibrium, it must be the case that all messages of equal cost or less are used in equilibrium.

Lemma 4 Consider $m^{i}$ which is transmitted with cost $c(i)$. Under Condition $L$, if $b=0$ and $m^{i}$ is used in equilibrium then every $m^{j}$ where $c(j) \leq c(i)$ is also used in equilibrium.

Proof: Suppose that there is an equilibrium $\left(\mu^{*}, \alpha^{*}\right)$ such that $\mu^{*}(s)=m^{i}$ with cost $c(i)$ however there does not exists an $s^{\prime}$ such that $\mu^{*}\left(s^{\prime}\right)=m^{j}$ and $c(j) \leq c(i)$. If the signal $m^{j}$ is observed, $R$ believes that the state is certain to be $s=0$. On the interval in the state space for which the $S$ sends message $m^{i}, S$ 's payoff cannot be higher than $-c(i)$. By Lemma 10, $S$ has identical payoffs at each of the states for which expression (4) is satisfied, including the states 0 and 1 . Therefore, at $s=0$, the sender has a payoff of less than $-c(i)$ and a profitable deviation is then to send $m^{j}$. Therefore, $\left(\mu^{*}, \alpha^{*}\right)$ cannot constitute an equilibrium.

We compare Lemma 2 with Lemma 4. According to Lemma 2, Condition $P$ implies that we are only guaranteed that the empty message will be used in every equilibrium. By contrast, Lemma 4 shows that each equilibrium under Condition $L$ implies that if a message of complexity $k$ is used in equilibrium then all other messages of complexity $k$ or less will also be used in equilibrium. An implication of this is that for $b=0$ we can denote an equilibrium by the most complex message used. Therefore, if the most complex equilibrium messages have $k$ elements then the number of costly equilibrium messages $(n)$ is:

$$
n=\sum_{j=1}^{k} \gamma^{j}
$$

Since we can denote an equilibrium by its most costly message used in equilibrium, if this message has a cost of $c(k)$ then we will refer to the equilibrium as a $k$-equilibrium.

Definition $3 A$-equilibrium is one in which all messages which cost less than or equal to $c(k)$ are used.

\footnotetext{
${ }^{16}$ There can be problems if an out-of-equilibrium message leads to beliefs other than $s=0$. For instance, when $b>0$ there are parameter values such that we are not guaranteed existence if an out-of-equilibrium message implies beliefs of $s=1$.
} 
We now show that we are guaranteed a $k$-equilibrium such that there does not exist a $k^{\prime}$-equilibrium for $k^{\prime}>k$. If such a $k$ is found then we say that the $k$-equilibrium is maximal. We now show that we are guaranteed a maximal equilibrium.

If a $k$-equilibrium is to exist then, given costs $c(0), c(1), \ldots, c(k-1), c(k)$ it must be that expressions (1), (3) and (4) are satisfied. Also, for an equilibrium to exist under Condition $P$ it must be that there are no holes.

Definition $4 A(\mu, \alpha)$ is L-feasible if it satisfies expressions (1), (3), (4) and additionally there does not exist an unused message $m^{k}$ and a used message $m^{k^{\prime}}$ such that $k \leq k^{\prime}$.

We now show that if preferences are aligned then for any amount of communication costs there is an $L$-feasible $(\mu, \alpha)$ which is maximal.

Lemma 5 If $b=0$ then for any $c>0$ there is always exists a maximal, L-feasible $(\mu, \alpha)$.

Proof: To check whether a $(\mu, \alpha)$ is $L$-feasible we rewrite expressions (3) and (4). There are $\gamma^{k}$ messages which cost $c(k)$ and are sent on an interval of size $\lambda^{k}$. There are $\gamma^{k-1}$ messages which cost $c(k-1)$ and are sent on an interval of size $\lambda^{k-1}=\sqrt{4[c(k)-c(k-1)]+\left(\lambda^{k}\right)^{2}}$. There are $\gamma^{k-2}$ messages which cost $c(k-2)$ and are sent on an interval of size $\lambda^{k-2}=$ $\sqrt{4[c(k)-c(k-2)]+\left(\lambda^{k}\right)^{2}}$. There are $\gamma^{2}$ messages which cost $c(2)$ and are sent on a interval of $\lambda^{2}=\sqrt{4[c(k)-c(2)]+\left(\lambda^{k}\right)^{2}}$. There are $\gamma$ messages which cost $c(1)$ and are sent on an interval of size $\lambda^{1}=\sqrt{4[c(k)-c(1)]+\left(\lambda^{k}\right)^{2}}$. Finally for the costless message, we write $\lambda^{0}=\sqrt{4 c(k)+\left(\lambda^{k}\right)^{2}}$. Therefore, we may write expression (3) as

$$
\begin{gathered}
\sqrt{4 c(k)+\left(\lambda^{k}\right)^{2}}+\gamma \sqrt{4[c(k)-c(1)]+\left(\lambda^{k}\right)^{2}}+\gamma^{2} \sqrt{4[c(k)-c(2)]+\left(\lambda^{k}\right)^{2}}+ \\
\ldots+\gamma^{k-2} \sqrt{4[c(k)-c(k-2)]+\left(\lambda^{k}\right)^{2}}+\gamma^{k-1} \sqrt{4[c(k)-c(k-1)]+\left(\lambda^{k}\right)^{2}}+\gamma^{k} \lambda^{k}=1 .
\end{gathered}
$$

When communication costs increase, $\lambda^{k}$ must decrease to zero in order for expression (6) to hold. Recall that we required that $c(i+1)-c(i) \geq \psi>0$ for all $i \in\{0, \ldots, k\}$. Therefore, we can write the lower bound of each term in the left hand side of expression (6):

$$
\sqrt{4 k \psi}+\gamma \sqrt{4(k-1) \psi}+\gamma^{2} \sqrt{4(k-2) \psi}+\ldots+\gamma^{k-2} \sqrt{4(2) \psi}+\gamma^{k-1} \sqrt{4 \psi}>1 .
$$

For every $\gamma$ and $\psi$, there is a $k$ large enough so that expression (7) is satisfied. Therefore, we are guaranteed a maximal, $L$-feasible $(\mu, \alpha)$.

Intuitively, Lemma 5 shows that, for any communication costs there exists an upper bound on the number of messages used in an equilibrium. It should not come as a surprise that full communication is not attained when $c>0$. However, the straightforward characterization of equilibrium under Condition $L$ is perhaps surprising, given the complicated nature of the equilibrium under Condition $P$. 
We are now ready for the main result of the section. Proposition 2 shows that an equilibria under Condition $L$ always exists. Further, the only equilibria under Condition $L$ are the ones in which are maximal among the strategies which which satisfy the necessary conditions.

Proposition 2 If $b=0$ then under Condition $L$ an equilibrium $\left(\mu^{*}, \alpha^{*}\right)$ always exists and it is exclusively a member of the maximal, L-feasible class.

Proposition 2 shows that under Condition $L$, only the equilibria with the largest possible number equilibrium messages does not have a profitable deviation. The proposition uses the language class because when $b=0$, the ordering of the set of messages does not matter. Also, one can see the full force of Condition $L$ by noting the difference between the multiplicity of equilibria in Propositon 1 and the uniqueness found in Proposition 2

As stated earlier, our restriction to pure strategies on the part of the sender is not without loss of generality. This is due to the fact that the proof of Proposition 2 crucially depends on this restriction. To see this, consider a maximal, $L$-feasible $(\mu, \alpha)$ for which there are states on which $S$ mixes between say two messages. In this case, Condition $L$ does not offer a profitable deviation and therefore we are not guaranteed a maximal equilibrium.

Our Proposition 2 is reminiscent of Proposition 3 in Chen, Kartik and Sobel (2008). The authors show that in the CS model where monotonicity holds, NITS admits only the most informative equilibrium. In the notation of our model, Chen, Kartik and Sobel show that for $b>0$ and $c=0$ in the uniform-quadratic case, NITS uniquely selects the most informative equilibrium. Our Proposition 2 and Proposition 3 of Chen, Kartik and Sobel becomes more surprising when we provide an example which demonstrates that we are not guaranteed uniqueness when $b>0$ and $c>0$.

\subsection{Simple Characterization}

Here we focus on the case where preferences are perfectly aligned $(b=0)$ and communication costs are linear in the complexity of the message. For instance, this will allow us to discuss the outcomes if the message space is $\varnothing \cup\left(\cup_{k=1}^{\infty}\{0,1,2,3,4,5,6,7,8,9\}^{k}\right)$. Suppose that $b=0$ and $c(k)=c k$. One might be tempted to conclude that an equilibrium of the following form might exist, $\mu(s)=d$ for $d \in\{0,1,2,3,4,5,6,7,8,9\}$ if $s \in[0.1 d, 0.1(d+1))$. However, even under the permissive Condition $P$, Lemma 2 shows that this cannot be an equilibrium because the empty message is not used. Further, if $c=0.1$ then the equilibrium under Condition $L$ is such that $\lambda^{0}=0.633$ and $\lambda^{1}=0.0366$.

Another benefit of this exercise is that, for general communication costs, it is difficult to characterize the threshold level of costs which render a $(\mu, \alpha) L$-feasible. However in the linear case, the characterization is rather simple. If $c(k) \leq c^{*}(k)$ then a $(\mu, \alpha)$ associated with a $k$-equilibrium is $L$-feasible and if $c(k)>c^{*}(k)$ then such a $(\mu, \alpha)$ is not $L$-feasible.

Lemma 6 If $c(k)=c k$ and $c>0$ then the cutoff cost for a $k$-equilibrium is:

$$
c^{*}(k)=\left(\frac{1}{2 \sum_{j=1}^{k} \gamma^{k-j} \sqrt{j}}\right)^{2} .
$$


Proof: At the largest $c$ such that signal $k$ is $L$-feasible, it must be that $\left(\lambda^{k}\right)^{2}=0$. By expression (5) it must be that, $\left(\lambda^{k-1}\right)^{2}=4 c,\left(\lambda^{k-2}\right)^{2}=8 c, \ldots,\left(\lambda^{1}\right)^{2}=4(k-1) c,\left(\lambda^{0}\right)^{2}=4 k c$. Therefore, we may write expression (6) in the case of linear costs as

$$
2 \sqrt{c(k)}+2 \gamma \sqrt{c(k-1)}+2 \gamma^{2} \sqrt{c(k-2)}+\ldots+2 \gamma^{k-2} \sqrt{c(2)}+2 \gamma^{k-1} \sqrt{c(1)}=1
$$

and so the lemma is proved.

In order to provide some intuition for the characterization to this point, we provide the following example. Given linear communication costs, the example illustrates that the equilibria under Condition $P$ possesses a large amount of multiplicity however Condition $L$ leaves only the most informative class of equilibria. The example also illustrates the utility of Lemma 6 .

Example 1 Consider the case where $c(i)=0.01 i, b=0$ and $\gamma=1$. Note that:

$$
c^{*}(4)=0.00662<0.01<c^{*}(3)=0.0145 .
$$

Therefore, under Condition $P$ there are four classes of $k$-equilibria ${ }^{17}$ where $k \in\{0,1,2,3\}$. Any $(\mu, \alpha)$ associated with a $k^{\prime}$-equilibria such that $k^{\prime} \geq 4$ is neither $P$-feasible nor L-feasible. For the $k=0$ equilibrium, $m^{0}$ gets sent on all states. For the $k=1$ case, there are two equilibria. There is an equilibrium where $m^{0}$ is sent on states $[0,0.52)$ and $m^{1}$ on states $[0.52,1]$. There is another equilibrium where $m^{1}$ is sent on states $[0,0.48)$ and $m^{0}$ on states $[0.48,1]$. Note that in each of the $k=1$ equilibria $\lambda^{0}=0.52$ and $\lambda^{1}=0.48$. For the $k=2$ case, there are six equilibria. There is a monotonic equilibria where $m^{0}$ is sent on states $[0,0.392), m^{1}$ on states $[0.392,0.729)$ and $m^{2}$ on $[0.729,1]$. The remaining 5 equilibria require that $\lambda^{0}=0.392, \lambda^{1}=0.337$, and $\lambda^{2}=0.271$. For the $k=3$ case, there are 24 equilibria. There is a monotonic equilibria where $m^{0}$ is sent on states $[0,0.363), m^{1}$ on states $[0.363,0.665), m^{2}$ on $[0.665,0.892)$ and $m^{3}$ on $[0.892,1]$. The remaining 23 equilibria require that $\lambda^{0}=0.363, \lambda^{1}=0.302, \lambda^{2}=0.227$ and $\lambda^{3}=0.108$. For Condition L, only the $24, k=3$ equilibria exist.

\section{Imperfect Alignement of Preferences under Condition $L$}

Recall Proposition 2 which demonstrated that the only equilibria under Condition $L$ are the ones which are maximal and $L$-feasible. Similarly, Proposition 3 in Chen, Kartik and Sobel (2008) shows that in the CS model where monotonicity holds, NITS admits only the most informative equilibrium. In the notation of our model, Chen, Kartik and Sobel show that for $b>0$ and $c=0$ in the uniform-quadratic case that NITS uniquely selects the most informative equilibrium.

For the case of $b=0$ and $c>0$, the order of the messages did not matter as long as their size was governed by expression (5). For the case of $b>0$ and $c=0$, the order of the

\footnotetext{
${ }^{17}$ This example is not exhaustive, in that there are also many equilibria under Condition $P$ which are not $k$-equilibria. For instance, there is an equilibrium where $m^{0}$ and $m^{i}$ are transmitted, where $i \in\{1, \ldots, 24\}$. There is another equilibrium where $m^{0}, m^{1}$ and $m^{j}$ are transmitted, where $j \in\{2, \ldots, 15\}$.
} 
signals themselves did matter; what did matter was that the intervals on which each message was sent were increasing. However, when $b>0$ and $c>0$ there is an interaction between these two effects, which might cause the nonuniqueness which we now describe. Note that this nonuniqueness can manifest itself in two distinct ways. First, there could exist several equilibria with a given set of equilibrium messages, however these equilibria differ in their informativeness. Second, there can exist equilibria which satisfy Condition $L$ yet differ in the set of equilibrium messages. The following example demonstrates this second aspect and the subsequent example demonstrates the first.

Example 2 Suppose that $b=0.245, \gamma=1$ and communication costs are $c(i)=0.01 i$. First, there exists an equilibrium $\left(\mu^{*}, \alpha^{*}\right)$ where two messages are used. Message $m^{0}$ is sent on $s \in[0,0.03)$ and the $m^{1}$ is sent on $s \in[0.03,1]$. The sender's $s=0$ equilibrium payoffs are $-(0.015-0.245)^{2}=-0.0529$, which is greater than deviation payoffs of $-(0.245)^{2}-0.02$ $=-0.080$. There also exists an equilibrium where $m^{0}$ is sent for all states. The sender's $s=0$ equilibrium payoffs are $-(0.5-0.245)^{2}=-0.065$, which is greater than deviation payoffs of $-(0.245)^{2}-0.01=-0.070$.

This example stands in contrast to Chen, Kartik and Sobel (2008) who find that NITS uniquely selects only the most informative equilibrium when $b>0$ and $c=0$. This example also stands in contrast to our Proposition 2 in that Condition $L$ uniquely selects only the most informative equilibria when $b=0$ and $c>0$.

Finally, as we show in the following example, when $b>0$ there exists equilibria where an increase in communication costs will improve communication.

Example 3 First, consider the costless communication case. When $b=0.2, \gamma=1$ and $c(i)=0$, there is only one outcome equivalent equilibria of the following form: a single action is induced on $s \in[0,0.1)$ and a single action is induced on $s \in[0.1,1]$. Message $m_{0}$ induces $a=0.05$ and message $m_{1}$ induces $a=0.55$. In this case, $E\left[-(a-s)^{2}\right]=-0.0608$. However, when $b=0.2, \gamma=1$ and $c(i)=0.01 i$, there are two non-outcome equivalent equilibria. In the first equilibria, $m^{0}$ is sent on $s \in[0,0.12)$ and $m^{1}$ on $s \in[0.12,1]$. In the second equilibria, $m^{1}$ is sent on $s \in[0,0.08)$ and $m^{0}$ on $s \in[0.08,1]$. In the first equilibria, $E\left[-(a-s)^{2}\right]=-0.0569$ and in the second, $E\left[-(a-s)^{2}\right]=-0.0649$. If the cost of communication is increased to $c(i)=0.02 i$ then in the first equilibria $m^{0}$ is sent on $s \in[0,0.14)$ and $m^{1}$ on $s \in[0.14,1]$, implying $E\left[-(a-s)^{2}\right]=-0.0532$. By way of comparison note that the equilibrium in which there is no communication implies $E\left[-(a-s)^{2}\right]=-0.0833$.

The above provides an example where an increase in communication costs can lead to an improvement in communication. Also note that Example 3 contained an instance of two distinct equilibria, which share the set of equilibrium messages yet differ in their informativeness. Finally, note that the last equilibrium described in Example 3 outperforms the upper bound for payoffs as found by Goltsman et. al. (2009). ${ }^{18}$ These authors find that the upper bound for the expected payoffs of the receiver in mediated communication is:

$$
E\left[-(a-s)^{2}\right]=-\frac{1}{3} b(1-b)=-0.0533 .
$$

\footnotetext{
${ }^{18}$ This possibility was first suggested by Andreas Blume.
} 
What is the intuition behind the equilbrium which outperforms the Goltsman et. al. (2009) upper bound? Note that there are two effects at work. When $b>0$, the sender increases the intervals at the upper end of the state space, which reduces the expected payoff to the receiver. However, the communication costs induce the sender to decrease the interval sizes on which the costly signal is sent. In the relevant equilibrium, the costly message is in the upper end of the state space. Therefore, these effects work in opposite directions, thereby achieving an expected payoff above that of the upper bound for the case where communication is not costly.

\section{Conclusions}

We have modeled an interaction between an informed sender and an uninformed receiver where communication is costly and discrete. We have characterized the equilibria under a permissive out-of-equilibrium restriction, Condition $P$. When sender and receiver have aligned preferences over the action of the receiver, we have demonstrated that under Condition $L$ only the most informative class of equilibria exists. This result is analogous to the application of the No Incentive to Seperate (NITS) condition to the uniform-quadric version of Crawford and Sobel (1982). Finally, for the case that preferences are not aligned, we have provided an example where Condition $L$ does not identify a unique equilibrium and that an increase in communication costs might improve communication. Further, this improvement can be large enough so that it outperforms the Goltsman et. al. (2009) upper bound on costless communication.

There remain interesting questions which are unanswered. Also, we have modeled the interaction as a single repetition. However, we are interested to learn the equilibrium behavior where the interaction is repeated. There are three possibilities as the relationship is potentially finitely repeated, infinitely repeated or is repeated until the communication attains some threshold. An additional issue which arises only in the repeated version of the game relates to learning on the part of the receiver. Presumably there is a relationship between some publicly observable signal and the optimal action for the receiver and also that the sender wishes to teach the receiver this relationship. Additionally, we are eager to learn the significance of our assumption of quadratic preferences and a uniform probability distribution. Finally, we are interested to know whether an environment with several heterogenous senders and receivers, would produce a novel matching problem.

We are currently working on a version of our model where the sender imperfectly observes the state. Our preliminary results, consistent with Blume, Board and Kawamura (2007), suggest that a small amount of this noise can improve communication.

Finally, we are eager to test our theoretical results in an experimental setting. Like most communication games, our equilibrium is quite complicated and this fact makes experimental investigation difficult. On the other hand, experimental papers have found suitable simplifications of the theoretical communication papers which they test. ${ }^{19}$

\footnotetext{
${ }^{19}$ For instance, Cai and Wang (2006) and Kawagoe and Takizawa (2009). Also see Blume et. al. (1998) and Blume et. al. (2001).
} 


\section{Appendix}

The appendix is organized as follows. First we prove a few results about the nature of the equilibria. Then we prove the results which appear in the body of the paper. Lemma 7 now shows that the intervals must be connected. Lemma 8 shows that the intervals cannot partially overlap. Then, we prove Lemma 1 . We prove these results for the case of mixed strategies. We will denote such possibly mixed strategies as $\widetilde{\mu}:[0,1] \rightarrow \Delta \mathcal{M}$.

Lemma 7 In any equilibria it cannot be the case that $m \in \widetilde{\mu}^{*}(\underline{s})=\widetilde{\mu}^{*}(\bar{s}), m^{\prime} \notin \widetilde{\mu}^{*}(\underline{s})=\widetilde{\mu}^{*}(\bar{s})$, $m^{\prime} \in \widetilde{\mu}^{*}\left(s^{\prime}\right)$ and $m \notin \widetilde{\mu}^{*}\left(s^{\prime}\right)$ where $\underline{s}<s^{\prime}<\bar{s}$.

Proof: Suppose there exists $m$ such that $\left(\widetilde{\mu}^{*}\right)^{-1}(m)=\left(s_{1}, s_{2}\right) \cup\left(s_{3}, s_{4}\right)$ with $\left(s_{1}, s_{2}\right) \cap$ $\left(s_{3}, s_{4}\right)=\emptyset$ and $\left(\widetilde{\mu}^{*}\right)^{-1}\left(m^{\prime}\right)=\left(s_{2}, s_{3}\right)$.

If $\bar{a}(m)=\bar{a}\left(m^{\prime}\right)$ and $c(m) \neq c\left(m^{\prime}\right)$ then there exists a profitable deviation for $S$ in choosing the cheaper message. If $\bar{a}(m)=\bar{a}\left(m^{\prime}\right)$ and $c(m)=c\left(m^{\prime}\right)$ then $m^{\prime} \in \tilde{\mu}^{*}(\underline{s})=\tilde{\mu}^{*}(\bar{s})$ and $m \in \widetilde{\mu}^{*}\left(s^{\prime}\right)$.

Now suppose that $\bar{a}(m) \neq \bar{a}\left(m^{\prime}\right)$. If $\bar{a}(m)<\bar{a}\left(m^{\prime}\right)$ and $m^{\prime} \in \tilde{\mu}^{*}(s)$ for $s \in\left(s_{2}, s_{3}\right)$ as

$$
-(\bar{a}(m)-s-b)^{2}-c(m)<-\left(\bar{a}\left(m^{\prime}\right)-s-b\right)^{2}-c\left(m^{\prime}\right) \text { for } s \in\left(s_{2}, s_{3}\right)
$$

then $m^{\prime} \in \widetilde{\mu}^{*}(s)$ for $s \in\left(s_{3}, s_{4}\right)$ as

$$
-(\bar{a}(m)-s-b)^{2}-c(m)<-\left(\bar{a}\left(m^{\prime}\right)-s-b\right)^{2}-c\left(m^{\prime}\right) \text { for } s \in\left(s_{3}, s_{4}\right) .
$$

If $\bar{a}(m)<\bar{a}\left(m^{\prime}\right)$ and $m \in \widetilde{\mu}^{*}(s)$ for $s \in\left(s_{3}, s_{4}\right)$ as

$$
-(\bar{a}(m)-s-b)^{2}-c(m)>-\left(\bar{a}\left(m^{\prime}\right)-s-b\right)^{2}-c\left(m^{\prime}\right) \text { for } s \in\left(s_{3}, s_{4}\right)
$$

then $m \in \widetilde{\mu}^{*}(s)$ for $s \in\left(s_{2}, s_{3}\right)$ as

$$
-(\bar{a}(m)-s-b)^{2}-c(m)>-\left(\bar{a}\left(m^{\prime}\right)-s-b\right)^{2}-c\left(m^{\prime}\right) \text { for } s \in\left(s_{2}, s_{3}\right) .
$$

The proof for the case of $\bar{a}(m)>\bar{a}\left(m^{\prime}\right)$ follows in the analogous manner.

Lemma 8 In any equilibria it cannot be the case that $m^{\prime} \in \widetilde{\mu}^{*}\left(s^{\prime}\right)$ where $s^{\prime} \in\left[s_{1}, s_{3}\right)$ and $m^{\prime \prime} \in \widetilde{\mu}^{*}\left(s^{\prime \prime}\right)$ where $s^{\prime \prime}=\left[s_{2}, s_{4}\right)$ where $s_{2}<s_{3}$.

Proof: Suppose that there was such an equilibrium. The message $m^{\prime}$ induces action $a^{\prime}$ and message $m^{\prime \prime}$ induced action $a^{\prime \prime}$. Therefore the payoff from sending $m^{\prime}$ is

$$
U^{R}\left(m^{\prime}\right)=-\left(a^{\prime}-s-b\right)^{2}-c\left(m^{\prime}\right)
$$

and the payoff from sending message $m^{\prime \prime}$ is

$$
U^{R}\left(m^{\prime \prime}\right)=-\left(a^{\prime \prime}-s-b\right)^{2}-c\left(m^{\prime}\right) .
$$

For $a^{\prime} \neq a^{\prime \prime}$ there is only a single state for which

$$
U^{R}\left(m^{\prime}\right)=U^{R}\left(m^{\prime \prime}\right)
$$


and therefore this cannot be the case. For $a^{\prime}=a^{\prime \prime}$ because $s_{2}<s_{1}<s_{3}<s_{4}$ and $c\left(m^{\prime}\right)<$ $c\left(m^{\prime \prime}\right)$ then there exists a profitable deviation by the sender to select the cheaper message. Therefore there cannot exist such an equilibrium.

Proof of Lemma 1: If there are $n+1$ distinct actions induced by the sender then it must be that there are $n$ equations in expression (2). If this was not the case then Definition 1 would not hold. A typical such expression would be the cutoff state between intervals such that $m_{h}^{i} \in \widetilde{\mu}^{*}\left(s^{\prime}\right)$ for $s^{\prime} \in\left[s_{h}, s_{h+1}\right), m_{h+1}^{j} \in \widetilde{\mu}^{*}\left(s^{\prime \prime}\right)$ for $s^{\prime \prime} \in\left[s_{h+1}, s_{h+2}\right)$ :

$$
-\left(\frac{s_{h}+s_{h+1}}{2}-s_{h+1}-b\right)^{2}-c(i)=-\left(\frac{s_{h+1}+s_{h+2}}{2}-s_{h+1}-b\right)^{2}-c(j) .
$$

Which we rewrite as:

$$
\begin{aligned}
-\left(\frac{s_{h}-s_{h+1}}{2}-b\right)^{2}-c(i) & =-\left(\frac{s_{h+2}-s_{h+1}}{2}-b\right)^{2}-c(j) \\
-\left(\frac{-\lambda_{h}^{i}}{2}-b\right)^{2} & =-\left(\frac{\lambda_{h+1}^{j}}{2}-b\right)^{2}+c(i)-c(j)
\end{aligned}
$$

so that

$$
\left(\lambda_{h+1}^{j}\right)^{2}-\left(\lambda_{h}^{i}\right)^{2}=4[c(i)-c(j)]+4 b\left(\lambda_{h}^{i}+\lambda_{h+1}^{j}\right) .
$$

Lemma 7 showed that the intervals must be connected. Lemma 8 showed that the equilibria cannot be partially overlapping. Either it is the case that the intervals have no states in common or the intervals completely overlap. Lemma 1 showed the relative size of the intervals as a function of their position on the state space and the cost of message. In the case that the intervals completely overlap, it must be that the communication cost of each message is identical, otherwise Lemma 1 would be violated. Note that we proved the above three lemmas for the case of mixed strategies, however the same results would hold if we restricted attention to pure strategies. In fact, as the next lemma shows, restricting attention to pure strategies under Condition $P$ can be done without loss of generality.

Lemma 9 Any distribution of outcomes in an equilibrium $\left(\widetilde{\mu}^{*}, \alpha^{*}\right)$ with mixed strategies under Condition $P$ can be replicated with an equilibrium $\left(\mu^{\prime *}, \alpha^{\prime *}\right)$ with pure strategies under Condition P.

Proof: Consider an equilibrium with mixed strategies $\left(\widetilde{\mu}^{*}, \alpha^{*}\right)$ and an equilibrium without mixed strategies $\left(\mu^{\prime *}, \alpha^{\prime *}\right)$ which generate identical outcomes. For the intervals on which the sender mixes, it must be that the messages have identical communication costs. Therefore, $\mu^{\prime *}$ is such that only one of those messages is used whereas the others are not. Further, under Condition $P$ those unused messages cannot constitute a profitable deviation for the sender because the action induced by the message would not be preferred to that attained in $\left(\mu^{* *}, \alpha^{*}\right)$

Note that the proof of Lemma 9 crucially depends on Condition $P$. It is not the case that the restriction to pure strategies under Condition $L$ is without loss of generality. 
Proof of Lemma 3: By assumption, there exits a $P$-feasible configuration of messages $\left(m_{L}, m_{R}\right)$ forming $(\mu, \alpha)$. Therefore, it must be that

$$
\lambda_{l+1}^{j}=\frac{4[c(i)-c(j)]}{\lambda_{l}^{i}+\lambda_{l+1}^{j}}+4 b+\lambda_{l}^{i}
$$

for $l \in\{1, \ldots, h-1\} \cup\{h+2, \ldots, n-1\}$ in addition to:

$$
\lambda_{h+1}^{j}=\frac{4[c(i)-c(j)]}{\lambda_{h-1}^{i}+\lambda_{h+1}^{j}}+4 b+\lambda_{h+1}^{i} .
$$

Now find the cheapest message in $\mathcal{M}$ which has not been used by either $m_{L}$ or $m_{R}$, say $m^{y}$. First, we check whether $\left(m_{L}, m_{h}^{y}, m_{R}\right)$ is $P$-feasible. In order to check this, it must be the case that

$$
\lambda_{l+1}^{j}=\frac{4[c(i)-c(j)]}{\lambda_{l}^{i}+\lambda_{l+1}^{j}}+4 b+\lambda_{l}^{i}
$$

for $l \in\{1, \ldots, n-1\}$ such that $\lambda_{h}^{y}$. If $\left(m_{L}, m_{h}^{y}, m_{R}\right)$ is not $P$-feasible then $\left(m_{L}, m_{R}\right)$ is the maximal, $P$-feasible configuration. If $\left(m_{L}, m_{h}^{y}, m_{R}\right)$ is $P$-feasible then we will find, among the available messages not used by either $m_{L}$ or $m_{R}$, the next most complex message, say $m^{z}$. Now we check whether $\left(m_{L}, m_{h}^{z}, m_{R}\right)$ is $P$-feasible. In order to check this, it must be the case that

$$
\lambda_{l+1}^{j}=\frac{4[c(i)-c(j)]}{\lambda_{l}^{i}+\lambda_{l+1}^{j}}+4 b+\lambda_{l}^{i}
$$

for $l \in\{1, \ldots, n-1\}$ such that $\lambda_{h}^{z}$. If $\left(m_{L}, m_{h}^{z}, m_{R}\right)$ is not $P$-feasible then $\left(m_{L}, m_{h}^{y}, m_{R}\right)$ is the maximal, $P$-feasible configuration. If $\left(m_{L}, m_{h}^{z}, m_{R}\right)$ is $P$-feasible then we will find, among the available messages not used by either $m_{L}$ or $m_{R}$, the next most complex message. We repeat this procedure until we have found an available $m^{k^{\prime}}$ such that $\left(m_{L}, m_{h}^{k^{\prime}}, m_{R}\right)$ is not $P$-feasible.

We are guaranteed such a $k^{\prime}$ because when increasing the complexity of the $h t h$ message, it must be that:

$$
\lambda_{l+1}^{j}=\frac{4[c(i)-c(j)]}{\lambda_{l}^{i}+\lambda_{l+1}^{j}}+4 b+\lambda_{l}^{i}
$$

for $l \in\{1, \ldots, n-1\}$. As the complexity of the message is increased, $\lambda_{h}^{j}$ must decrease. To see this note that $[c(i)-c(j)]$ decreases due to the assumption that $c(j)-c(j-1) \geq \psi>0$ for all $j$. For large enough $j$ the term $[c(i)-c(j)]$ becomes negative. Additionally, the term $\lambda_{h-1}^{i}+\lambda_{h}^{j}$ is bounded by 1 . Hence, there must be a $k^{\prime}$ such that $\left(m_{L}, m_{h}^{k^{\prime}}, m_{R}\right)$ is not feasible. Therefore, there exists a there exists a maximal, $P$-feasible configuration $\left(m_{L}, m_{h}^{k}, m_{R}\right)$ forming equilibria $\left(\mu^{*}, \alpha^{*}\right)$

Proof of Proposition 1: Suppose that $(\mu, \alpha)$ is $P$-feasible. Given $\alpha$, there does not exist a profitable deviation from $\mu$ regarding the messages used in equilibrium since it satisfies expression (4). There does not exist a profitable deviation for $S$ by sending an outof-equilibrium message since there are no new actions induced by such a message. Given $\mu$ which satisfies Lemma 1 there is no profitable deviation for $R$ from $\alpha$ since it satisfies expression (1). Therefore, $(\mu, \alpha)$ is an equilibrium. 
Lemma 10 If $b=0$ and there are $n$ actions induced then there are $n+1$ solutions to $\max _{s}\left(\bar{a}\left(m^{\prime}\right)-s^{\prime}\right)^{2}+c\left(m^{\prime}\right)$.

Proof: Suppose that $U^{S}(\bar{a}, \widehat{m}, \underline{s})>U^{S}(\bar{a}, \widehat{m}, \bar{s})$ where $\mu([\underline{s}, \bar{s}))=\widehat{m}$. As the distribution is uniform, $\bar{a}=\frac{\underline{s}+\bar{s}}{2}$. This implies that $\left(\frac{\underline{\underline{s}}+\bar{s}}{2}-\underline{s}\right)^{2}>\left(\frac{\underline{\underline{s}}+\bar{s}}{2}-\bar{s}\right)^{2}$, which cannot be the case. Combined with expression (2), we have $n+1$ such solutions

Hence, if $b=0$ and there are $n$ actions induced there are $n+1$ states with the worst ex-post payoff. Naturally these are candidates for reasonable beliefs in the event of an outof-equilibrium message. Further, any of these $n+1$ states would be sufficient for the results under Condition $L$ to hold.

Proof Proposition 2: First we show that an equilibrium under Condition $L$ always exists. As Lemma 5 shows, there will always be a maximal, $L$-feasible $(\mu, \alpha)$. Suppose that expression (6) is satisfied for $k$. We need to check that it is not profitable for the sender such that $s=0$, to transmit message a message of complexity $k+1$. Because $k$ satisfies expression (6) it must be that $\lambda^{0}=\sqrt{4 c(k)+\left(\lambda^{k}\right)^{2}}$. By Lemma 10, the equilibrium payoffs for the $S$ who received signal $s=0$ is:

$$
-\left(\frac{\lambda^{0}}{2}-0\right)^{2}-c(0)=-\left(\frac{\lambda^{1}}{2}-0\right)^{2}-c(1)=\ldots=-\left(\frac{\lambda^{k}}{2}-0\right)^{2}-c(k) .
$$

All of the messages used in equilibrium will not provide a profitable deviation, therefore we must use an out-of-equilibrium message to find a deviation. Any deviation accomplished by message of complexity $k+x$ where $x>1$ can be accomplished with a lower communication cost by sending message of complexity $k+1$. Therefore, the cheapest (and therefore best candidate) out-of-equilibruim message is the message with complexity $k+1$. If such a message is sent, $R$ would have beliefs that the message was sent by state $s=0$. Sending this signal yields a payoff of $-c(k+1)$. Therefore, the signal will be profitable when $\left(\lambda^{k}\right)^{2}>4[c(k+1)-c(k)]$.

For the case that $\lambda^{k+1}=0$ it must also be that $\lambda^{k}=4[c(k+1)-c(k)]$. However, when $k+1$ is not $L$-feasible it must be that $\left(\lambda^{k}\right)^{2}<4 c(k)$ and there is no profitable deviation. Therefore, when $k$ is $L$-feasible and $k+1$ is not, there is no profitable deviation to an equilibrium with a signal more complex than $k$ and so a $k$-equilibrium always exists.

Now we will show that if $k$ is $L$-feasible then there does not exist an equilibrium in which the most complex signal is $k^{\prime}$ where $k^{\prime}<k$. Suppose that $k$ is $L$-feasible and $k+1$ is not. Consider a candidate $k^{\prime}$-equilibrium. This candidate equilibrium is characterized by:

$$
\begin{gathered}
\left(\widehat{\lambda}^{j}\right)^{2}-\left(\widehat{\lambda}^{i}\right)^{2}=4(c(i)-c(j)) \text { for } i, j \in\left\{1, . ., k^{\prime}\right\} \\
\widehat{\lambda}^{k^{\prime}}>0 \\
\widehat{\lambda}^{0}+\widehat{\lambda}^{1}+\ldots+\widehat{\lambda}^{k^{\prime}}=1
\end{gathered}
$$


When $\lambda^{k}=0$, a $k$-equilibrium would require expression (3) to hold where $\lambda^{k}=0$ and $\lambda^{k^{\prime}}=$ $4\left[c(k)-c\left(k^{\prime}\right)\right]$. When $\lambda^{k}>0$ it must be that

$$
\left(\lambda^{k^{\prime}}\right)^{2}=4\left[c(k)-c\left(k^{\prime}\right)\right]+\left(\lambda^{k}\right)^{2}>4\left[c(k)-c\left(k^{\prime}\right)\right] .
$$

Therefore, it must be that $\left(\hat{\lambda}^{k^{\prime}}\right)^{2}>4\left[c(k)-c\left(k^{\prime}\right)\right]$ and that $\left(\hat{\lambda}^{0}\right)^{2}>4 c(k)$. So we can write the equilibrium payoffs as:

$$
U^{S}=-\left(\frac{\left(\widehat{\lambda}^{0}\right)^{2}}{2}-0\right)^{2}<-c(k)
$$

Deviation payoffs are $-c(k)$, therefore equilibrium payoffs are less than deviation payoffs and so a $k^{\prime}$-equilibrium cannot exist.

To see that each $k$-equilibria uniquely determines the values of $\lambda$, we can rewrite expression (3) as:

$$
\begin{aligned}
& 2 \sqrt{c(z)+\left(\lambda^{k}\right)^{2}}+2 \gamma \sqrt{c(z)-c(1)+\left(\lambda^{k}\right)^{2}}+2 \gamma^{2} \sqrt{c(z)-c(2)+\left(\lambda^{k}\right)^{2}}+\ldots \\
& +2 \gamma^{z-1} \sqrt{c(z)-c(z-2)+\left(\lambda^{k}\right)^{2}}+2 \gamma^{z} \sqrt{c(z)-c(z-1)+\left(\lambda^{k}\right)^{2}}+\lambda^{k}=1
\end{aligned}
$$

The left hand side of expression (8) is strictly increasing in $\lambda^{k}$ and therefore must only hold for a single value of $\lambda^{k}$. And so the proposition is proved. 


\section{References}

Austen-Smith, David (1994), "Strategic Transmission of Costly Information." Econometrica, 62(4), 955-963.

Austen-Smith, David and Banks, Jeffrey (2000), "Cheap Talk and Burned Money." Journal of Economic Theory, 91, 1-16.

Banks, Jeffrey and Sobel, Joel (1987), "Equilibrium Selection in Signalling Games." Econometrica, 55, 647-661.

Blume, Andreas and Board, Oliver (2009), "Language Barriers." Working paper University of Pittsburgh.

Blume, Andreas, Board, Oliver and Kawamura, Kohei (2007), "Noisy talk." Theoretical Economics, 2, 395-440.

Blume, Andreas, DeJong, Douglas, Kim, Yong-Gwan and Sprinkle, Jeffrey (1998), "Experimental evidence on the evolution of meaning of messages in sender-reciever games." American Economic Review, 88, 1323-1340.

Blume, Andreas, DeJong, Douglas, Kim, Yong-Gwan and Sprinkle, Jeffrey (2001), "Evolution of Communication with Partial Common Interest." Games and Economic Behavior, 37, $79-120$.

Cai, Hongbin and Wang, Joseph Tao-Yi (2006), "Overcommunication in strategic information transmission games." Games and Economic Behavior, 56, 7-36.

Calvo-Armengol, Antoni, de Marti, Joan and Prat, Andrea (2009), "Endogenous Communication in Complex Organizations." Working paper, Autonoma de Barcelona, Pompeu Fabra and London School of Economics.

Che, Yeon-Koo and Kartik, Navin (2009), "Opinions as Incentives." Journal of Politial Economy, 117(5), 815-860.

Chen, Ying, Kartik, Navin and Sobel, Joel (2008), "Selecting Cheap-Talk Equilibria." Econometrica, 76(1), 117-136.

Cho, In-Koo and Kreps, David (1987), "Signaling Games and Stable Equilibria." Quarterly Journal of Economics, 102, 179-221.

Crawford, Vincent and Sobel, Joel (1982), "Strategic Information Transmission." Econometrica, 50(6), 1431-1451.

Cremer, Jacques, Garicano, Luis and Prat, Andrea (2007), "Language and the Theory of the Firm." Quarterly Journal of Economics, 117, 373-407.

Dewatripoint, Mathias and Tirole, Jean (2005), "Modes of Communication." Journal of Political Economy, 113(6), 1217-1238. 
Farrell, Joseph (1993), "Meaning and Credibility in Cheap-Talk Games." Games and Economic Behavior, 5, 514-531.

Fischer, Paul and Stocken, Phillip (2001), "Imperfect Information and Credible Communication." Journal of Accounting Research, 39(1), 119-134.

Goltsman, Maria, Horner, Johannes, Pavlov, Gregory and Squintani, Francesco (2009), "Mediation, Arbitration and Negotiation." Journal of Economic Theory, 144, 1397-1420.

Gossner, Olivier, Hernandez, Penelope and Neyman, Abraham (2006), "Optimal Use of Communication Resources." Econometrica, 74(6), 1603-1636.

Jager, Gerhard, Koch-Metzger, Lars and Riedel, Frank (2009), "Voronoi Languages: Equilibria in Cheap-Talk Games with High-Dimensional Types and Few Signals." Working paper Bielefeld University.

Kartik, Navin (2007), "A Note on Cheap Talk and Burned Money." Journal of Economic Theory, 136, 749-758.

Kawagoe, Toshiji and Takizawa, Hirokazu (2009), "Equilibrium refinement vs.level-k analysis: An experimental study of cheap-talk games with private information." Games and Economic Behavior, 66, 238-255.

Kohlberg, Elon and Mertens, Jean-Francois (1987), "On the Strategic Stability of Equilibria." Econometrica, 54, 1003-1037.

Lipman, Barton (2006), "Why is Language Vague?" Working paper, Boston University.

Matthews, Steven, Okuno-Fujiwara, Masahiro and Postlewaite, Andrew (1991), "Refining Cheap-Talk Equilibria." Journal of Economic Theory, 55, 247-273.

Morgan, John and Stocken, Phillip (2003), "An Analysis of Stock Recommendations," RAND Journal of Economics, 34(1), 183-203.

Morris, Steven (2001), "Political Correctness." Journal of Political Economy, 109(2), 231265.

Spector, David (2000), "Pure Communication Between Agents with Close Preferences." Economics Letters, 66, 171-178.

Spence, Michael (1973), "Job Market Signaling." Quarterly Journal of Economics, 87(3), 355-374.

Vartiainen, Hannu (2009), "A Simple Model of Secure Public Communication." Theory and Decision, 67, 101-122.

Verrecchia, Robert (1983), "Discretionary disclosure." Journal of Accounting and Economics, 5, 179-194.

Verrecchia, Robert (2001), "Essays on Disclosure." Journal of Accounting and Economics, 32, 97-180. 